\title{
An Algorithm Combining Latent Dirichlet Allocation and Bimodal Network for Evaluating Goal Deviation of Intellectual Property Strategy Execution in China
}

\author{
Bing Sun, ${ }^{1}$ Mingxing Yu, ${ }^{1}$ and Zaoli Yang $\mathbb{C}^{2}$ \\ ${ }^{1}$ School of Economics and Management, Harbin Engineering University, Harbin 150001, China \\ ${ }^{2}$ College of Economics and Management, Beijing University of Technology, Beijing 100124, China \\ Correspondence should be addressed to Zaoli Yang; yangzaoli@hotmail.com
}

Received 3 October 2020; Revised 2 November 2020; Accepted 7 November 2020; Published 21 November 2020

Academic Editor: Shouzhen Zeng

Copyright (c) 2020 Bing Sun et al. This is an open access article distributed under the Creative Commons Attribution License, which permits unrestricted use, distribution, and reproduction in any medium, provided the original work is properly cited.

China has implemented the intellectual property strategy since 2008 to support innovation-driven development. However, statistical data issued during the "12th Five-Year Plan" (2011-2015) showed that there are certain deviations between the actual and expected intellectual property strategy's goals. To effectively diagnose the goal deviation, an algorithm combining the latent Dirichlet allocation and bimodal network based on policy text was proposed. In this method, topics in intellectual property policy texts of China's provincial regions were extracted through the latent Dirichlet allocation model, and a bimodal network centered at provincial administration district-policy topics was constructed. Subsequently, the characteristics of the goal execution deviation of the IPS in the provincial government were explored based on the centrality of the bimodal network and singular value decomposition. Finally, some diagnosis results and conclusions were demonstrated to provide reasonable methods for evaluation of national strategic planning and promoting policy performance.

\section{Introduction}

China's economic development is characteristic of decreasing growth rate, the urgent needs of structural upgrading, and dynamic transformation since 2008. To get rid of resource restrictions, realize the transformation from resource element-driven development to innovation-driven development, and finally improve the economic development quality, the Chinese government issued the Outline of National Intellectual Property Strategy in 2008, which symbolized the application of intellectual property strategy (IPS) in innovation-driven development, to eliminate resource restrictions, transform resource element-driven development to innovation-driven development, and improve the economic development quality. IPS has become the overall strategy that has driven China to the knowledgebased economic development in the coming decades [1]. The central and provincial regions enacted more than 500 policy measures and documents for the smooth implementation of the IPS from 2009 to 2017. Hence, the IPS system under Chinese policies and laws was formed gradually. However, a large gap between the actual and expected goals of Chinese IPS was observed. In 2016, China released the National Intellectual Property Protection and Application Planning during the "13th Five-Year Plan," which indicated that Chinese intellectual property (IP) during the "12th FiveYear Plan" still faces numerous challenges, such as incoordination between the number and quality of intellectual properties, the small number of core patents, famous brands and high-quality copyrights, poor association with the market, low transformation rate of achievements, and imperfect management mechanism, as well as backward awareness of intellectual property protection, which restrict the improvement of China's intellectual property protection capability. The successful implementation of China's national overall strategy relies highly on the execution of provincial regions' supporting policy measures, which is the main reason for the deviation between the actual and 
expected goals of Chinese IPS. Nevertheless, existing studies mainly focus on evaluating the IPS or other public policies' goal performance [2-7] rather than analyzing the deviation characteristics from the execution stages of provincial regions. Thus, they neither accurately evaluate the specific execution stage of the overall goal deviation of the national IPS nor disclose the causes of deviation. From this perspective, diagnosing the goal execution deviation of Chinese IPS in the provincial region's implementation stage, accurately positioning relevant weak stages, scientifically adjusting the implementation of the strategic plan, and assuring maximized implementation benefits are practically crucial.

The remainder of this paper is organized as follows. Section 3 introduces the methodologies, which mainly include data source and processing, LDA topic mining model, and bimodal network analysis. Section 4 describes the empirical analysis of the goal execution deviation characteristics of IPS in China's provincial regions based on policy texts. Section 5 presents the results and discussions. Section 6 draws the conclusions and proposes the future prospects. The framework of this study is shown in Figure 1.

\section{State of the Art}

Studies on the diagnosis and evaluation of IPS under the traditional paradigm can be divided into two types. First, a diagnosis or evaluation model was constructed on the basis of the goal-driven evaluation paradigm by using the open structural file information of public departments to evaluate the implementation effect of the IPS. Song et al. established a set of evaluation index systems and methods to measure the IP creation, IP application, IP protection, and IP management based on collected data in the statistical report of intellectual properties in China's scientific research institutions [2]. Additionally, statistical data related to patents were acquired to construct the patent application network model [3] and a "three stages-two dimensions" model [4]. These models were used to evaluate the patent value or quality and to diagnose the implementation effect of the IPS indirectly. Alternatively, the capability of patent industrialization was evaluated and the commercial effect of the IPS was diagnosed by using the collected transaction information in the technological market through the fuzzy multiattribute comprehensive evaluation method [5] and the equation of patent standardized measurement [8]. Second, an econometric model was constructed on the basis of the theoretical hypothesis-driven diagnosis paradigm to test the present theoretical hypothesis by using data samples collected from field investigation or by summarizing statistical data of the document. Most of the associated studies focused on the influences of IPS or IP policies on product output [9-12] and innovation level [13, 14] or on enterprises' technological innovation $[15,16]$ and production efficiency [17-19]. However, the traditional evaluation models, which are based on the structural information of single-file data and driven by goals and theoretical hypothesis, can only diagnose the implementation results of IPS. National strategies or public policies cover multiple stages, such as the formation, execution, evaluation, feedback, and correction of public policies [20]. The traditional goal- and theoretical hypothesis-driven evaluation models based on structural information have a weak information basis. Therefore, the final evaluation results cannot involve the diagnosis of most policy execution stages or others.

Different from the traditional evaluation modes, the data-driven evaluation for policy effectiveness has become a new paradigm and been practiced in education policy $[21,22]$, energy policy [23-25], safety management [26, 27], building [28, 29], public medical policy [30-32], and so on. However, the aforementioned studies only achieve the local perfection of public policies on specific occasions with limited structured information; they have not discussed execution-stage evaluation problems that involve public policy processes. With the continuous development of the Internet in recent years, the public-derived big data based on the Internet unstructured text information may exert reconstructive influences on the national decision-making process [33]. The public policy evaluation driven by unstructured text information has started. However, existing associated studies only theoretically discuss the feasibility of such public policy evaluation and conduct a qualitative analysis of the influences of Internet unstructured text information on policy formulation and legislation changes [34] as well as the optimization of public policies [33]. Internet unstructured text information has not been applied in the field of public policy evaluation. Relevant studies remain in the theoretical exploration stage and associated empirical analysis must be extended urgently.

The goal of strategic planning is an important component of the national strategy and public policy [35]. The gap between the overall execution and expected goals has been expanding since China implemented the IPS in 2008. However, studies on the goal deviation of IPS, let alone goal execution deviation, are limited. Organizational behavioral science concerned "goal deviation" early. This field described goal deviation as follows: the organization deviates from the original preset goal and shifts to other goals in the actual process due to the influences of different factors [36]. Subsequently, connotations of goal deviation were further extracted. Representative scholars Resh and Marvel indicated that goal deviation occurs when the organization focuses on the replacement of output indexes, which are difficult to measure and have great relevance [37]. Liu et al. argued that goal deviation is an organizational phenomenon that pays further attention to the easy-to-measure explicit goal or uses the mean as the goal rather than the preset goal [38].

The study on the goal execution deviation of IPS can only be screened indirectly from previous investigations. For example, Wang and Hsieh [5] and Tamura [8] evaluated the application of IP and its commercial performance. The goal execution deviation is gained indirectly on the basis of a simple comparison between the evaluation results and the expected goal of IPS, but the characteristic of goal execution deviation remains difficult to obtain. As the goal of IPS is realized by specific policies and measures of the provincial region, the research scale on the goal execution deviation of 


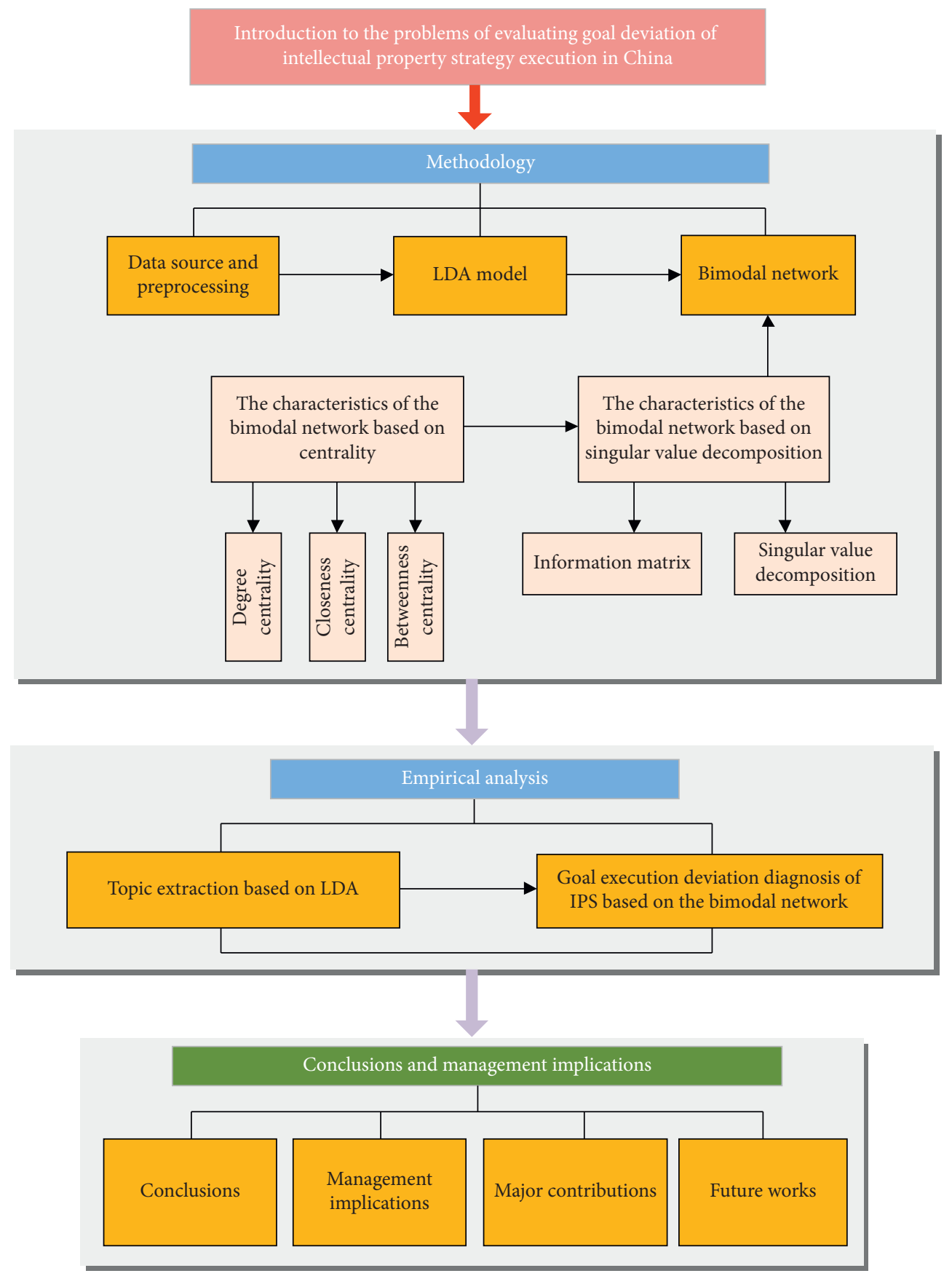

FIgURE 1: The framework of this study.

IP policies, which are important components of public policies, has been formed, thus laying the foundation for the goal execution deviation diagnosis. However, the goal execution deviation diagnosis of public policies sets different measurable variables, including the incompletion degree of the goal and the fuzzy degree of results 35 [39-42]. Subsequently, the degree of goal execution deviation is determined through empirical analysis based on a questionnaire survey or statistical data. Research perspectives remain limited in the implementation outcome of public policies due to the structured information, and the specific execution has not been discussed yet.
Overall, previous studies on goal execution deviation diagnoses of IPS and relevant policies are mainly based on the traditional goal- and theoretical hypothesis-driven paradigm. Diagnosis results only display the achievement of the overall goal but neglect the specific execution of policies. Moreover, the information source is limited in the secondhanded data of government statistical report, and heterogeneous information lacks analysis based on policy texts, which hinder decision-makers from identifying multiple causes of goal execution deviation of IPS. For this reason, considering the advantages of the LDA model for accurately extracting text topics, the LDA model was introduced to 
mine topics in the IP policy text of provincial regions. Then, since the bimodal network can analyze the relationship between two kinds of heterogeneous things, a bimodal network centered at topics and provinces in IP policy text was constructed. Through this network, the goal execution deviation of provincial regions was diagnosed in accordance with the structural characteristics of the bimodal network.

\section{Methodology}

3.1. Data Source and Preprocessing. In this study, the text files of policies, plans, and strategic plans related to IP from 2008 to 2017 were downloaded through artificial interpretation from IP websites of selected 30 provincial regions and of the Department of Science and Technology in China. A total of 449 effective policy texts were selected after the release of the Outline of National Intellectual Property Strategy in 2017 with considerations to the regionality and integrity of policy texts, which covered more than 220,000 Chinese characters.

The 449 original policy texts were transformed into the common CSV format, marked with provinces. IP policy texts also have specific writing norms. By combining the word-formation rules of the noun phrase and formal standards of policy texts, subject terms in policy texts were extracted in accordance with part-of-speech tagging and the extraction rule of noun phrases. This approach includes constructing a new stop words library (which is applicable to IP policy texts based on the stop words library of Harbin Institute of Technology) and adding empty words (which are inapplicable for extracting notional subject terms from the stop words library), such as "middle-level," "11th Five-Year Plan," and "copies." In addition, subject terms were extracted through the Jieba Chinese word segmentation. During the extraction, the dictionary named dict.txt in Jieba was used and a noun dictionary on IP policy analysis was constructed. For example, some professional terms in the IP policy text were added to the new dictionary, such as "IP creation," "IP use or application," "IP protection," "patent," and "trademark." Subject terms in policy texts were selected through Jieba word segmentation in Python, thus transforming an IP policy text into the eigenvectors comprising several subject terms about IP. These eigenvectors were used in the subsequent topic model analysis.

3.2. LDA Model. LDA is a statistical topic model constructed by Blei [43] for extracting potential topics. The basic principle of LDA is to calculate the conditional distribution of potential variables under the given observation variables in accordance with a joint probability distribution. The recognition method of potential document contents, which is based on the Bayes probability of words, topics, and documents, is typical unsupervised learning.

In the LDA model, documents were viewed as the mixed probability distribution of potential subjects, and topics were viewed as the probability of several words. Therefore, potential topics and documents were regarded as the probability distributions of vocabulary and potential topics, respectively, which is beneficial for projecting the document into the topic layer on a large scale. Figure 2 shows the process.

In Figure $1, \theta$ and $Z$ are implicit variables, and $W$ denotes the observable vocabulary variable. A document set $D=\left\{d_{1}, d_{2}, \ldots, d_{m}\right\}$ covers $m$ documents and each document $d$ contains $n$ words. The document word set is $d=\left\{w_{1}, w_{2}, \ldots, w_{n}\right\}$. As assumed, $D$ is extracted into $T$ topics and $T_{k}$ represents the $k$-th potential topic. The specific process is introduced as follows [43]:

(1) The Dirichlet distribution with a $\beta$ parameter is calculated for $T$-dimensional vector $Z$, $Z \sim \operatorname{Dirichlet}(\beta)$.

(2) The Dirichlet distribution with a $\alpha$ parameter is derived for $T$-dimensional vector $\theta$, $Z \sim \operatorname{Dirichlet}(\alpha)$.

(3) With respect to vocabulary $w$ in the document: the multinominal distribution with a $\theta$ parameter is generated for topic $z$, and $P(w \mid z, \beta)$ is obtained for the vocabulary in accordance with the $\beta$ parameter:

$$
P(w \mid z, \beta)=\int p(\theta \mid \alpha)\left(\prod_{i=1}^{n} \sum_{k=1}^{T} P\left(z_{k} \mid \theta\right) P\left(w_{i} \mid z_{k}, \beta\right)\right) \mathrm{d} \theta
$$

where $\theta$ and $Z$ are implicit variables and $W$ denotes the observation variable. The LDA model calculates the posterior and conditional distributions of implicit variables under the given observable document.

3.3. Bimodal Network. A bimodal network is formed by two types of heterogeneous nodes through certain connections. The dataset in the bimodal network is the entity that represents different types by rows and columns and is mainly utilized to describe the "binary" structural relationship between a group of actors and the active events. In this study, a bimodal network that uses provincial and policy topics as the heterogeneous nodes was constructed by extracting relevant topics from all IP policy texts of provincial government departments through LDA. Subsequently, the goal execution deviation of the national IPS of provincial regions was reflected by the structural characteristics of the bimodal network.

In the bimodal network, the two types of heterogeneous nodes are actors and events [44]. In this study, actors and events represent the provinces and policy topics of IP, respectively. The characteristic indexes of the bimodal network are as follows.

3.3.1. The Characteristics of the Bimodal Network Based on Centrality. The node centrality of the bimodal network is a quantitative analysis of individual power, which explores the positional characteristics of all nodes in the network from the perspective of microscopic nodes [45]. This model 


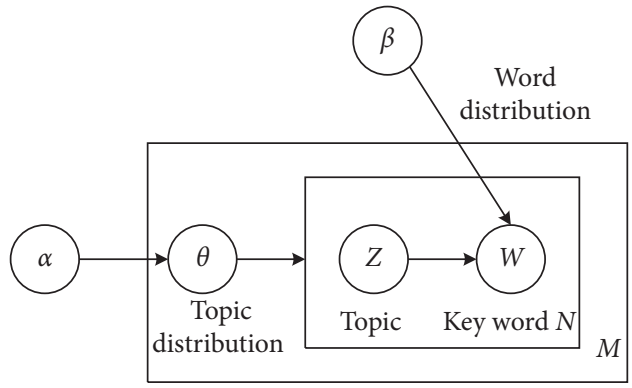

Figure 2: Principle of the LDA topic model.

mainly includes degree, closeness, and betweenness centralities.

(1) Degree Centrality. Degree centrality refers to the degree of connections between the actor and other actors in the network. The actor with more connections has a stronger influence than that with fewer connections [45]. The degree centrality of provincial nodes is the number of topics concerned by the provincial nodes, whereas the degree centrality of topic nodes refers to the number of involved provinces. If $g$ provinces form the actor set $n=\left(n_{1}, n_{2}, \ldots, n_{g}\right)$ and $h$ topics form the event set $z=\left(z_{1}, z_{2}, \ldots, z_{h}\right)$, then the degree centralities of province $i$ and topic $k$ can be expressed as

$$
\begin{aligned}
C_{D}^{\mathrm{NZ}}\left(n_{i}\right) & =\sum_{k=1}^{g+h} x_{i k}^{\mathrm{NZ}}, \quad(i=1,2, \ldots, g ; k=1,2, \ldots, h), \\
C_{D}^{\mathrm{NZ}}\left(z_{k}\right) & =\sum_{i=1}^{g+h} x_{i k}^{\mathrm{NZ}}, \quad(i=1,2, \ldots, g ; k=1,2, \ldots, h),
\end{aligned}
$$

where NZ is the bimodal network comprising $g$ provinces and $h$ topics. $x_{i k}^{\mathrm{NZ}}$ denotes the affiliation relationship data of the bimodal network of $g \times h$ bipartite matrix $x^{\mathrm{NZ}}$.

(2) Closeness Centrality. Closeness centrality is applied to represent the time for information diffusing from one node to another [44]. Different from closeness centrality in the single-mode network, the closeness centrality of actors in the bimodal network is proportional to the sum of the distance between one node and other nodes in the network and between the node and all event nodes. The closeness centrality of events is similar. The calculation formulas for the closeness centralities of actors and events are as follows:

$$
\begin{aligned}
& C_{C}^{\mathrm{NZ}}\left(n_{i}\right)=\left[1+\frac{\sum_{j=1}^{g+h} \min k d(k, j)}{g+h-1}\right]^{-1},(k=1,2, \ldots, h), \\
& C_{C}^{\mathrm{NZ}}\left(m_{k}\right)=\left[1+\frac{\sum_{i=1}^{g+h} \min j d(i, j)}{g+h-1}\right]^{-1},(i=1,2, \ldots, g) .
\end{aligned}
$$

In equation (3), connections exist between event point $k$ and actor $i$. In equation (4), actor $j$ and event node $k$ are connected. $d(k, j)$ indicates the distance between event node $k$ and node $j$ in the bimodal network. Here, $j$ can be used to represent the event or actor node.

(3) Betweenness Centrality. Betweenness centrality represents the positional importance of nodes in the network. In the bimodal network, connections between every two actor nodes must run through all event nodes concerning them [45]. Therefore, the event nodes are in the shortest route between actors. Similarly, actor nodes are in the shortest route between event nodes. For this reason, calculating the betweenness centrality of event nodes in the bimodal network must consider all actor nodes concerning the event node. The calculation formulas of the betweenness centrality of actor and event nodes are as follows:

$$
C_{B}^{\mathrm{NZ}}\left(n_{i}\right)=\frac{1}{2} \sum_{m_{k}, m_{l} \in n_{i}}^{g+h} \frac{1}{x_{k l}^{M}}, \quad(i=1,2, \ldots, g ; k=1,2, \ldots, h),
$$

$$
C_{B}^{\mathrm{NZ}}\left(m_{k}\right)=\frac{1}{2} \sum_{n_{i}, n_{j} \in m_{k}} \frac{1}{x_{i j}^{N}}, \quad(i=1,2, \ldots, g ; k=1,2, \ldots, h) .
$$

In equation (7), actors $n_{i}$ and $n_{j}$ concern a total of $x_{i j}^{N}$ events. For any pair of actors $\left(n_{i}, n_{j}\right)$ in $m_{k}$, the betweenness centrality contribution of $m_{k}$ is $1 / x_{i j}^{N}$ units. $x^{N}$ and $x^{M}$ represent the data matrixes of single nodes of actors and events, respectively.

3.3.2. The Characteristics of the Bimodal Network Based on Singular Value Decomposition (SVD). Singular value analysis is used to reduce the dimension of the bimodal network. The hierarchical classification of the network structure is conducted, and the classification factors hidden in the bimodal network are recognized. The network characteristics are close to the overall structure of the bimodal network by analyzing the hidden classification factors. The calculation principle of the SVD method is as follows [44].

An $n \times m$ information matrix of the bimodal network $X$ $(n \geq m)$ is available. Matrixes $U, D$, and $V$ are simultaneously obtained through SVD. $X=U \times D \times V^{\prime}$, where $V^{\prime}$ is the transposed matrix of $V$ and $D$ denotes the $r \times r$ diagonal matrix and has $r$ singular values. Matrixes $U$ and $V$ are $n \times r$ and $m \times r$ matrixes, which cover the $r$ eigenvector of matrix $X X^{\prime}$. The corresponding eigenvalues are arranged in descending order. A real matrix $A$ with an order of $r$ can be decomposed as follows:

$$
A=U\left[\left[\begin{array}{llll}
a_{1} & & & \\
& a_{2} & & \\
& & \ldots & \\
& & & a_{r}
\end{array}\right] V^{\prime} .\right.
$$

Equation (8) is defined as the SVD of matrix $A$. In equation (8), matrixes $U$ and $V I$ are orthogonal matrixes and 
$a_{i}>0(i=1,2, \ldots, r)$, where $a_{1}, a_{2}, \ldots, a_{r}$ is the $A$ singular value of the matrix. Dimensions of topics in IP policy texts are reduced by the bimodal network constructed through SVD. Topics are classified into factors with common characteristics, which is conducive to analysis loads of provincial topics on different factors. Hence, the preference of different provinces in knowledge property strategy is evaluated from the perspective of the overall network.

\section{Empirical Results and Discussions}

4.1. Topic Extraction Based on LDA. LDA modeling is performed through Gibbs sampling. Topics are analyzed by using Gensim source packages based on the Python software. Moreover, 4-20 topics and 1000 iterations are set, thereby obtaining the document-topic and word-topic probability matrixes according to formula (1). Topic clustering data under different numbers of topics are compared repeatedly. Finally, nine topics are selected in accordance with the nine task goals in Outline of National Intellectual Property Strategy. Table 1 lists the 10 major characteristic words of each topic.

Table 1 shows that nine subjects are selected on the basis of the LDA topic model, namely, IP culture, IP intermediary services, IP transformation and applications, IP legal system, IP administration management, IP overseas cooperation, IP talents, IP law enforcement, and IP creation. These topics are the key strategic goals proposed in the Outline of National Intellectual Property Strategy. The provincial topics are classified in accordance with previous marks of provinces, thereby further exploring the frequency distributions of each provincial policy text in the nine topics. Then, the number of topics involved in all policy texts of each province is calculated, which provides a data basis for the subsequent construction of the bimodal network matrix and explores contents hidden in the province-topic information matrix.

4.2. Goal Execution Deviation Diagnosis of IPS Based on the Bimodal Network. The bimodal data are visualized automatically by using Ucinet 6.0 software on the basis of the data matrix of the constructed bimodal network. Figure 2 presents the results.

Figure 3 intuitively displays the province-topic binary affiliation relationship. On the basis of the size of the topic center point, IP transformation and applications, IP administration management, IP overseas cooperation, and IP legal system are topics with the highest concern frequency of the provincial region, whereas IP culture and IP intermediary services are topics of less concern. This finding indicates the general emphasis of the provincial region to implement IPS.

Furthermore, the bimodal network forms the singleproperty concurrence network from the perspectives of topics and provinces to explore the single-property concurrence connotations (Figure 3).

The single-property concurrence network of topics in Figure 4(a) reflects two or more topics simultaneously concerned by provincial IP policy. On the basis of the thick connections of different topics, IP administration management, IP legal system, IP overseas cooperation, and IP creation are highly concerned by provincial regions in IPS implementation. The single-property concurrence network of topics in Figure 4(b) reflects the number of topics concerned by more than two provinces. Similarly, numerous topics are simultaneously concerned by Shanghai, Beijing, Tianjin, and Shanxi, but only a few topics are concerned by Hebei, Guizhou, and Ningxia.

The characteristics of nodes are further generated by using Ucinet 6.0. In Table 2, great hierarchical differences are observed among 30 provinces in terms of degree and closeness centralities. Beijing, Tianjin, Shanghai, Chongqing, Sichuan, Gansu, Qinghai, Hunan, and Guangdong rank the top positions in view of the degree centrality of provincial nodes. Hence, these provinces are concerned with extensive and comprehensive scopes of IP policy topics. The content scope and execution speed of supporting policies in these provinces are inferior to those of other provinces. With respect to closeness centrality, Beijing, Tianjin, Shanghai, Chongqing, and Sichuan occupy the first layer of the network, indicating their quick responses to the topics in national IP policies. This result implies that these provinces can formulate supporting policies in accordance with local situations immediately after the release of the national IPS. Nevertheless, all selected provinces show relatively similar betweenness centrality, indicating the unsubstantial performance as the bridge for information diffusion and transportation. This finding conforms to the actual situation of the bimodal network of policies. Data sources of the policy network of different provinces are mainly contributed by the number of concerns of topic information. The characteristics do not highlight the social affiliation. Hence, using provincial nodes as the bridge in the single-mode network is difficult.

In addition, the microstructural characteristics of topic nodes in Table 3 reflect that the three types of microscopic characteristics show a consistent variation trend. IP transformation and applications, IP legal system, IP administration management, and IP overseas cooperation show the highest degree centrality. This result shows that provincial region's supporting policies provide the highest concerns on the five topics. Topics ranking the top of closeness centrality also belong to the five topics, which confirms that these topics are easy to mention and emphasize through local supporting policies. This finding is closely related to the subsequent successive release of details and directed optimal adjustment of the central government. Similarly, the five topics have the highest betweenness centrality, indicating the prominent universal effect among IP transformation and applications, IP legal system, IP administration management, and IP overseas cooperation. The five topics also serve as the "bridge" in the topic node network. When one or several provincial supporting policies concern these topics, it can be easily "followed" by other provinces. The "followers" may pay great attention to the related supporting policy contents of other provinces to a large extent when they formulate supporting policies. On the contrary, microstructural data of talents, IP law enforcement, IP creation, IP 
TABLe 1: Distribution of topics-characteristic words.

\begin{tabular}{|c|c|c|c|c|}
\hline Topics & IP culture & $\begin{array}{c}\text { IP intermediary } \\
\text { services }\end{array}$ & $\begin{array}{c}\text { IP transformation and } \\
\text { applications }\end{array}$ & IP legal system \\
\hline \multirow{9}{*}{ Characteristic words } & Culture & Intellectual property & Intellectual property & \multirow{3}{*}{$\begin{array}{c}\text { Intellectual property } \\
\text { Cases } \\
\text { Administrative } \\
\text { enforcement }\end{array}$} \\
\hline & Patents & Comments & Patents & \\
\hline & Trademark & Rights protection & Cultivation & \\
\hline & Request & Aids & $\begin{array}{c}\text { Scientific and technological } \\
\text { achievements }\end{array}$ & Rights protection \\
\hline & Review & Service & Scientific technology & \multirow{5}{*}{$\begin{array}{c}\text { Complaints } \\
\text { Open } \\
\text { Law enforcement } \\
\text { Infringement } \\
\text { Intellectual property } \\
\text { protection }\end{array}$} \\
\hline & Service & Patents & Pilot project & \\
\hline & Talents & Pledge & Industry & \\
\hline & Intellectual property & Copyright & Construction & \\
\hline & Rewards & Enterprises & Demonstrations & \\
\hline $\begin{array}{l}\text { IP administration } \\
\text { management }\end{array}$ & IP overseas cooperation & IP talents & IP law enforcement & IP creation \\
\hline Methods & Projects & Intellectual property & Intellectual property & Patents \\
\hline Standards & Patent technology & Popularization & Mediation & Patents \\
\hline Law enforcement & Industry & Talents & Law enforcement & Innovation \\
\hline Reward & Financial aid & Cultivation & Copyright & Patent technology \\
\hline Confirmation & Overseas & Scientific technology & $\begin{array}{l}\text { Administration and law } \\
\text { enforcement }\end{array}$ & Products \\
\hline Talent & Progress & Construction & Intellectual property protection & Inventor \\
\hline Patents & $\begin{array}{c}\text { Autonomous intellectual } \\
\text { property }\end{array}$ & Innovation & Impact & Design \\
\hline Intellectual property & Intellectual property office & Applications & Administration department & \multirow{2}{*}{$\begin{array}{c}\text { Knowledge property } \\
\text { Scientific and technologic } \\
\text { type }\end{array}$} \\
\hline Financial aid & Strategy & Engineering & Intellectual property office & \\
\hline
\end{tabular}

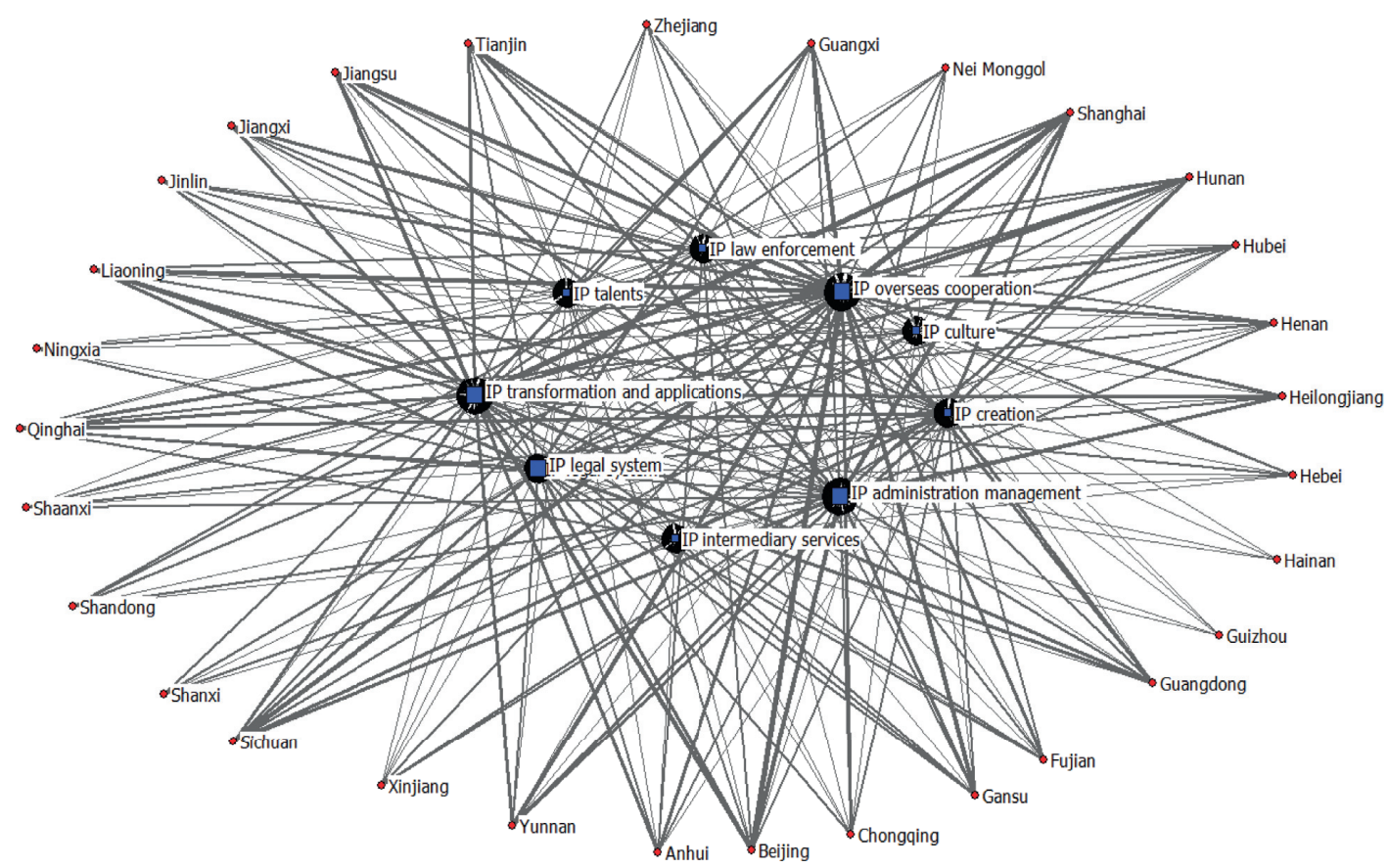

Figure 3: Visualized diagram of the province-topic bimodal network.

culture, and IP intermediary services are relatively small, especially the microstructural data of culture and intermediary services. The degree, closeness, and betweenness centralities of IP culture and IP intermediary services are lower than those of the seven remaining topics, which reflects less attention by provincial regions on these two topics. Moreover, the optimization efficiency and performance quantification of the two topics are considerably poorer than those of superior IP transformation and applications. This finding explains the weak positions of IP 
TABLE 2: Characteristics of provincial nodes.

\begin{tabular}{|c|c|c|c|}
\hline Province & Degree centrality & Closeness centrality & Betweenness centrality \\
\hline Beijing & 0.231 & 1.716 & 0.001 \\
\hline Tianjin & 0.231 & 1.716 & 0.001 \\
\hline Hebei & 0.179 & 1.62 & 0.001 \\
\hline Shanxi & 0.154 & 1.575 & 0 \\
\hline Nei Mongol & 0.154 & 1.575 & 0.001 \\
\hline Heilongjiang & 0.179 & 1.62 & 0.001 \\
\hline Jinlin & 0.205 & 1.667 & 0.001 \\
\hline Liaoning & 0.205 & 1.667 & 0.001 \\
\hline Shanghai & 0.231 & 1.716 & 0.001 \\
\hline Jiangsu & 0.205 & 1.667 & 0.001 \\
\hline Chongqing & 0.231 & 1.716 & 0.001 \\
\hline Sichuan & 0.231 & 1.716 & 0.001 \\
\hline Yunnan & 0.205 & 1.667 & 0.001 \\
\hline Shaanxi & 0.179 & 1.62 & 0.001 \\
\hline Gansu & 0.231 & 1.716 & 0.001 \\
\hline Qinghai & 0.231 & 1.716 & 0.001 \\
\hline Ningxia & 0.128 & 1.533 & 0 \\
\hline Xinjiang & 0.179 & 1.62 & 0.001 \\
\hline Hainan & 0.103 & 1.494 & 0 \\
\hline Guizhou & 0.128 & 1.533 & 0 \\
\hline Zhejiang & 0.205 & 1.667 & 0.001 \\
\hline Anhui & 0.205 & 1.667 & 0.001 \\
\hline Fujian & 0.205 & 1.667 & 0.001 \\
\hline Jiangxi & 0.205 & 1.667 & 0.001 \\
\hline Shandong & 0.154 & 1.575 & 0 \\
\hline Henan & 0.205 & 1.667 & 0.001 \\
\hline Hubei & 0.205 & 1.667 & 0.001 \\
\hline Hunan & 0.231 & 1.716 & 0.001 \\
\hline Guangdong & 0.231 & 1.716 & 0.001 \\
\hline Guangxi & 0.205 & 1.667 & 0.001 \\
\hline
\end{tabular}

TABle 3: Characteristics of topic nodes.

\begin{tabular}{lccc}
\hline Topic & Degree centrality & Closeness centrality & Betweenness centrality \\
\hline IP culture & 0.462 & 1.643 & 0.009 \\
IP intermediary services & 0.513 & 1.742 & 0.009 \\
IP transformation and applications & 0.744 & 2.396 & 0.024 \\
IP legal system & 0.718 & 2.3 & 0.022 \\
IP administration management & 0.744 & 2.396 & 0.024 \\
IP overseas cooperation & 0.769 & 2.5 & 0.027 \\
IP talents & 0.615 & 1.983 & 0.015 \\
IP law enforcement & 0.615 & 1.983 & 0.015 \\
IP creation & 0.692 & 2.212 & 0.02 \\
\hline
\end{tabular}

culture and IP intermediary services in the supporting policies of provincial regions for IPS.

The overall features of the province-topic bimodal network are further explored through the SVD, which is used for the hierarchical classification of topics and provinces. The interpretation capability of the first three factors accounted for $76.5 \%$, which is calculated by using Ucinet 6.0, indicating that these factors can interpret the macroscopic relation characteristics between provinces and topics. In addition, the SVD data of topic nodes are obtained, through which the topic factors are calculated as shown in Table 4.
Table 3 shows that characteristic factor 1 is a public factor, which indicates that the topic of characteristic factor 1 is used as the public and universal major content in provincial knowledge property policies. Data in Table 4 reflect that IP overseas cooperation and IP transformation and applications are public and universal topics highly concerned by local provinces in formulating the supporting IP policies. For factors 2 and 3, topics with high values in different factors are clustered into one type. In factor 2, IP culture, IP intermediary services, IP law enforcement, and IP creation are important topics and can be classified into the IP development environment. IP transformation and 
TABLE 4: SVD data of topic nodes.

\begin{tabular}{lccc}
\hline & Factor 1 & Factor 2 & Factor 3 \\
\hline IP culture & 0.052 & 0.153 & -0.14 \\
IP intermediary services & 0.077 & 0.189 & -0.181 \\
IP transformation and applications & 0.473 & -0.305 & 0.27 \\
IP legal system & 0.302 & 0.354 & 0.443 \\
IP administration management & 0.393 & -0.575 & -0.52 \\
IP overseas cooperation & 0.644 & 0.074 & 0.152 \\
IP talents & 0.102 & 0.085 & 0.206 \\
IP law enforcement & 0.129 & 0.092 & 0.072 \\
IP creation & 0.283 & 0.609 & -0.58 \\
\hline
\end{tabular}

applications, IP legal system, IP administration management, IP overseas cooperation, and IP talents are five topics belonging to factor 3 and are classified as the IP operation system.

On the basis of the classification of factors 2 and 3, SVD data of nodes in provinces were investigated to classify the policy topics issued by each province accurately (Table 5).

Table 5 exhibits that the supporting IP policies of Shanxi, Chongqing, Sichuan, Yunnan, Shaanxi, Gansu, Qinghai, Xinjiang, Henan, Fujian, Hunan, and Guangdong pay further attention to factor 2, that is, the IP development environment. On the contrary, other provinces pay further attention to IP operation system. With regard to loads of public factors, Beijing, Tianjin, Heilongjiang, Jilin, Shanghai, and Jiangsu rely on public factors. The supporting IP policies in these provinces attach further attention to IP overseas cooperation and IP transformation and applications.

For a further intuitive display of distributions of the three factors in different provinces, the relation visualization diagram between two eigenvectors comprising factor numerical values is illustrated to describe the eigenvalue relationship among different factors (Figure 5).

In Figure 4, the $x$-axis is factor 1 and the $y$-axis represents factors 2 and 3 . In factor 1, IP overseas cooperation, IP transformation and applications, and IP administration management are prominent topics and have the largest contribution to public factors. IP culture and IP intermediary services are easy to cluster (Figure 5(a)). Shanxi, Chongqing, and Guangdong are regions in the same dimensions. IP transformation and applications and IP legal system are easy to cluster (Figure 5(b)), which involves Beijing, Tianjin, and Hebei. The factor clusters of provinces reflected by relationships among eigenvalues of different factors agree with the province-topic clustering results of the SVD.

\section{Conclusions and Management Implications}

5.1. Conclusions. In this study, topics in IP policy texts of the provincial region were extracted by using the LDA model, and the goal execution deviation of the IPS of the provincial region was diagnosed by the bimodal network. The following conclusions can be drawn.

First, in the process of implementing national intellectual property strategic goals, provincial regions have paid much attention to topics such as IP transformation and applications, IP administration, IP overseas cooperation, IP creation, and IP legal system, whereas they have paid very little attention to topics such as IP culture, IP intermediary services, IP talents, and IP law enforcement. These overlooked topics present the provincial regions' execution deviation.

Second, when it comes to establishing the supporting policies, Beijing, Tianjin, Shanghai, Chongqing, Sichuan, Gansu, Qinghai, Hunan, and Guangdong provinces played the role as forerunners, while other provinces played the role as followers. It is also indicated that the topics that these forerunner governments have concerned about are more timely and comprehensive.

Third, the topics of IP transformation and applications, IP administration, IP overseas cooperation, IP creation, and IP legal system played the roles as "bridge" in the network. When the supporting policies of one or several provinces have paid attention to the above five topics, it is more likely to cause other provinces to focus on these five topics as well when establishing supporting policies. In contrast, the topics of IP talents, IP law enforcement, IP creation, IP culture, and IP intermediary services are at the edge of the network.

5.2. Management Implications. Based on the above findings, we offered the following management implications to correct provincial regions' IP strategy execution deviation.

First, when provincial regions implement the IP strategic goals, it is better to refer to the nine major goals in the Outline of National Intellectual Property Strategy. Provincial regions should make effort to balance the nine goals of IP culture, IP intermediary services, IP transformation and applications, IP legal system, IP administration, IP overseas cooperation, IP talents, and IP law enforcement in order to avoid execution deviation.

Second, provincial regions should learn rationally rather than blindly accepting and following other provinces. It is suggested that provincial regions can propose the corresponding policies by taking into consideration their regional and developmental phase characteristics.

Third, provincial regions should cooperate more often when implementing policies. They should learn from each other's experience and consider their own logical path of implementing the nine strategic goals. Therefore, the IP strategy execution deviation or omission can be prevented as much as possible. It is not only about improving the patent quality but also about commercial efficiency. Provincial regions should not only strengthen the legal system and law enforcement but also improve the environment and culture for IP creation and operation.

The major contributions of this paper are discussed as follows.

First, the combination of LDA topic mining and bimodal network can offset the shortcomings of traditional models that cannot diagnose nonstructured policy texts. This condition can reflect the goal deviation among different execution stages of the national strategic planning.

Second, the relation network of heterogeneous nodes, which cannot be constructed in the single-mode network, 


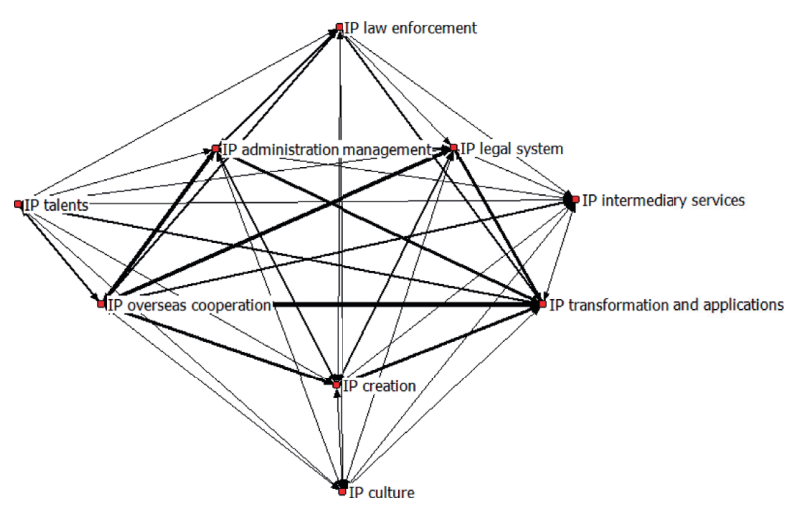

(a)

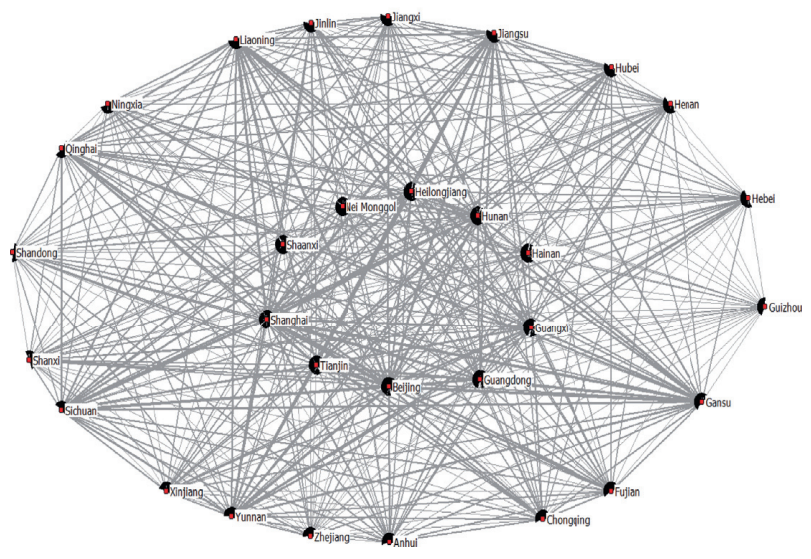

(b)

FIGURE 4: Single-property concurrence network generated by the bimodal network. (a) Single-property concurrence network of topics. (b) Single-property concurrence network of provinces.

TABLE 5: SVD data of nodes in provinces.

\begin{tabular}{|c|c|c|c|}
\hline Province & Factor 1 & Factor 2 & Factor 3 \\
\hline Beijing & 0.338 & -0.302 & 0.078 \\
\hline Tianjin & 0.204 & -0.068 & -0.053 \\
\hline Hebei & 0.085 & -0.045 & -0.001 \\
\hline Shanxi & 0.086 & 0.081 & 0.029 \\
\hline Nei Mongol & 0.073 & -0.107 & 0.115 \\
\hline Heilongjiang & 0.207 & -0.352 & -0.071 \\
\hline Jinlin & 0.126 & -0.244 & -0.047 \\
\hline Liaoning & 0.261 & -0.151 & 0.167 \\
\hline Shanghai & 0.353 & -0.286 & -0.129 \\
\hline Jiangsu & 0.245 & -0.138 & 0.116 \\
\hline Chongqing & 0.15 & 0.036 & -0.154 \\
\hline Sichuan & 0.246 & 0.334 & -0.227 \\
\hline Yunnan & 0.236 & 0.014 & -0.223 \\
\hline Shaanxi & 0.062 & 0.078 & -0.153 \\
\hline Gansu & 0.234 & 0.121 & -0.184 \\
\hline Qinghai & 0.191 & 0.467 & -0.208 \\
\hline Ningxia & 0.065 & 0.117 & 0.217 \\
\hline Xinjiang & 0.091 & 0.039 & -0.345 \\
\hline Hainan & 0.026 & -0.005 & -0.118 \\
\hline Guizhou & 0.022 & 0.007 & 0.089 \\
\hline Zhejiang & 0.07 & -0.061 & -0.049 \\
\hline Anhui & 0.167 & -0.042 & 0.25 \\
\hline Fujian & 0.156 & 0.086 & -0.068 \\
\hline Jiangxi & 0.167 & 0.202 & 0.337 \\
\hline Shandong & 0.092 & -0.057 & -0.038 \\
\hline Henan & 0.119 & -0.017 & 0.142 \\
\hline Hubei & 0.116 & 0.071 & 0.269 \\
\hline Hunan & 0.242 & 0.131 & -0.068 \\
\hline Guangdong & 0.212 & 0.22 & 0.013 \\
\hline Guangxi & 0.204 & 0.287 & 0.466 \\
\hline
\end{tabular}

can be realized on the basis of the LDA topic mining and bimodal network model. This relation network can reflect the roles of provincial administration regions and policy topics in the network, diagnose the accuracy of goal execution deviations, and accurately recognize key topics.
Third, "concurrence" and "marginality" of singleproperty nodes of policy topics can be measured accurately on the basis of the LDA topic mining and bimodal network model. The goal execution deviation is diagnosed objectively and comprehensively during the implementation of the 


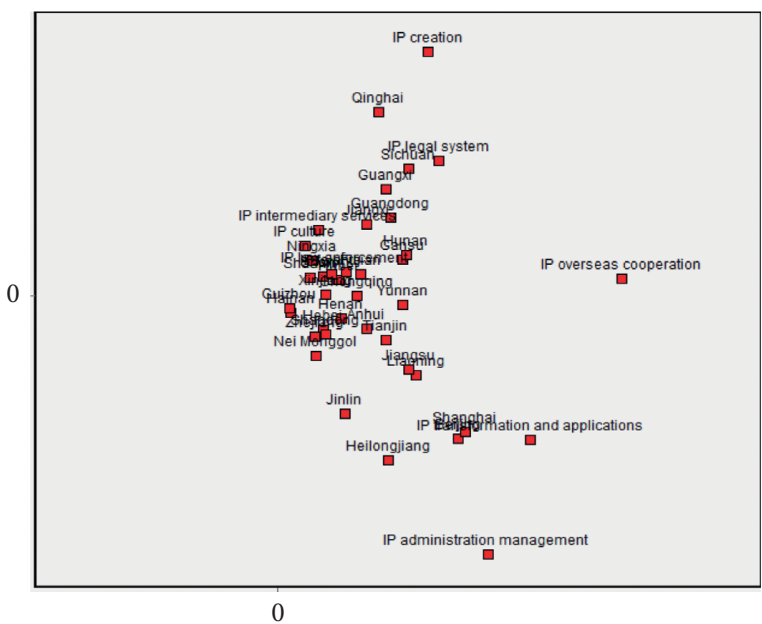

(a)

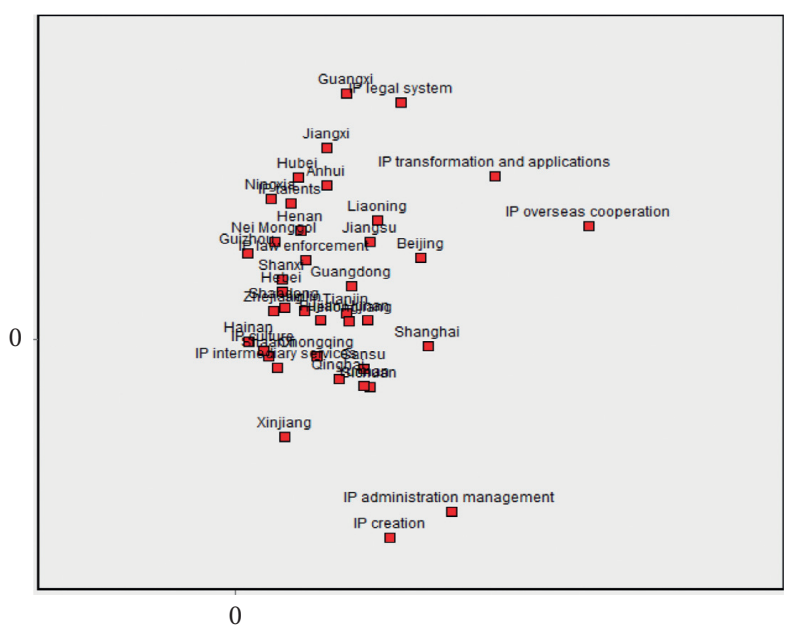

(b)

FIgURE 5: Analysis of singular values. (a) Relationship between factors 1 and 2. (b) Relationship between factors 1 and 3.

strategic plan by the provincial region. A simple and reliable method is offered.

The goal execution deviation for the Chinese national IPS is diagnosed by policy text information, and meaningful results are obtained. This study can further extend the research contents in the following aspects: (1) Popular topics of IP policies are analyzed by combining heterogeneous information on network media, forums, social software, and instant music video, which provides a further extensive basis for the goal execution deviation diagnosis. (2) The occurrence mechanism of deviation is comprehensively analyzed, which is beneficial for constructing the deviation correction mechanism.

\section{Data Availability}

All the data used to support the findings of this study are included within the article.

\section{Conflicts of Interest}

There are no conflicts of interest.

\section{Acknowledgments}

This research was supported by the Natural Science Foundation of China (Grant no. 71704007), the Beijing Social Science Foundation of China (Grant no. 18GLC082), and the Beijing Financial Foundation of China (Grant no. PXM2020-178216-000001).

\section{References}

[1] T. Dong, "On the relationship between the NIPS and Chinese economic development," Studies in Science of Science, vol. 5, pp. 641-652, 2009.

[2] H. F. Song, Y. Li, and W. Qu, "The intellectual property capacity measurement indicator system and method based on equivalent relationship of kinds of intellectual property and kinds of research institute," Studies in Science of Science, vol. 12, pp. 1826-1834+1825, 2013.

[3] G.-C. Yang, G. Li, C.-Y. Li et al., "Using the comprehensive patent citation network (CPC) to evaluate patent value," Scientometrics, vol. 105, no. 3, pp. 1319-1346, 2015.

[4] Y. T. Sun and Q. Luan, “A three stage-two dimensions' model of patent quality measuring and its empirical study: a case study of C9 league," Science of Science and Management of S.\&T.vol. 6, pp. 23-32, 2016.

[5] B. Wang and C.-H. Hsieh, "Measuring the value of patents with fuzzy multiple criteria decision making: insight into the practices of the Industrial Technology Research Institute," Technological Forecasting and Social Change, vol. 92, pp. 263-275, 2015.

[6] A. Flynn, "Investigating the implementation of SME-friendly policy in public procurement," Policy Studies, vol. 39, no. 4, pp. 422-443, 2018.

[7] L. Iain and B. B. Bella, "Distinctive policy diffusion patterns, processes and actors: drawing implications from the case of sport in international development," Policy Studies, vol. 39, no. 4, pp. 444-464, 2018.

[8] S. Tamura, "Effects of integrating patents and standards on intellectual property management and corporate innovativeness in Japanese electric machine corporations," International Journal of Technology Management, vol. 59, no. 3-4, pp. 180-202, 2016.

[9] P. Alfons, H. J. Paul, and W. Elizabeth, "The effect of patents on trade," Journal of International Economics, vol. 105, pp. 1-9, 2017.

[10] C. Mercedes and D. Marco, "Intellectual property rights, trade agreements, and international trade," Research Policy, vol. 48, no. 3, pp. 531-545, 2019.

[11] M. Lee, J. D. Alba, and D. Park, "Intellectual property rights, informal economy, and FDI into developing countries," Journal of Policy Modeling, vol. 40, no. 5, pp. 1067-1081, 2018.

[12] E. Billette de Villemeur, R. Ruble, and B. Versaevel, "Dynamic competition and intellectual property rights in a model of product development," Journal of Economic Dynamics and Control, vol. 100, pp. 270-296, 2019.

[13] B. Julia, C. Paolo, M. Lukas, and B. Kilian, "Intellectual property rights hinder sequential innovation. Experimental 
evidence," Research Policy, vol. 45, no. 10, pp. 2054-2068, 2016.

[14] A. C. Chu, H. Fan, G. Shen, and X. Zhang, "Effects of international trade and intellectual property rights on innovation in China," Journal of Macroeconomics, vol. 57, pp. 110-121, 2018.

[15] M. Grimaldi, M. Greco, and L. Cricelli, "A framework of intellectual property protection strategies and open innovation," Journal of Business Research, vol. 123, pp. 156-164, 2021.

[16] Z. Liu, R. Mu, S. Hu, L. Wang, and S. Wang, "Intellectual property protection, technological innovation and enterprise value-An empirical study on panel data of 80 advanced manufacturing SMEs," Cognitive Systems Research, vol. 52, pp. 741-746, 2018.

[17] R. Smeets and A. de Vaal, "Intellectual property rights and the productivity effects of MNE affiliates on host-country firms," International Business Review, vol. 25, no. 1, pp. 419-434, 2016.

[18] B. Kim, E. Kim, D. J. Miller, and J. T. Mahoney, “The impact of the timing of patents on innovation performance," Research Policy, vol. 45, no. 4, pp. 914-928, 2016.

[19] I. Nemlioglu, "A comparative analysis of intellectual property rights: a case of developed versus developing countries," Procedia Computer Science, vol. 158, pp. 988-998, 2019.

[20] L. Xue and Z. L. Lin, "Three perspectives to understand public policy process and their implications for China study," Chinese Public Administration, vol. 5, pp. 41-46, 2013.

[21] G. Alison and L. Bob, "NAPLAN data: a new policy assemblage and mode of governance in Australian schooling," Policy Studies, vol. 37, no. 6, pp. 568-582, 2016.

[22] E. Kurilovas, "On data-driven decision-making for quality education," Computers in Human Behavior, vol. 107, Article ID 105774, 2020.

[23] D. Lee, M. Kim, and J. Lee, "Adoption of green electricity policies: investigating the role of environmental attitudes via big data-driven search-queries," Energy Policy, vol. 90, pp. 187-201, 2016.

[24] X. Fu, G. Li, X. Zhang, and Z. Qiao, "Failure probability estimation of the gas supply using a data-driven model in an integrated energy system," Applied Energy, vol. 232, pp. 704-714, 2018.

[25] S. Zeng, X. Peng, T. Baležentis, and D. Streimikiene, "Prioritization of low-carbon suppliers based on Pythagorean fuzzy group decision making with self-confidence level," Economic Research-Ekonomska Istraživanja, vol. 32, no. 1, pp. 1073-1087, 2019.

[26] Z. L. Yang, H. Garg, J. Li, G. Srivastavad, and Z. Cao, "Investigation of multiple heterogeneous relationships using a q-rung orthopair fuzzy multi-criteria decision algorithm," Neural Computing and Applications, pp. 1-22, 2020.

[27] B. Wang, C. Wu, L. Huang, and L. Kang, "Using data-driven safety decision-making to realize smart safety management in the era of big data: a theoretical perspective on basic questions and their answers," Journal of Cleaner Production, vol. 210, pp. 1595-1604, 2019.

[28] Z. Yang, J. Roth, and R. K. Jain, "DUE-B: data-driven urban energy benchmarking of buildings using recursive partitioning and stochastic frontier analysis," Energy and Buildings, vol. 163, pp. 58-69, 2018.

[29] T. Ahmad, H. Chen, Y. Guo, and J. Wang, “A comprehensive overview on the data driven and large scale based approaches for forecasting of building energy demand: a review," Energy and Buildings, vol. 165, pp. 301-320, 2018.
[30] S. Burris, L. Hitchcock, J. Ibrahim, M. Penn, and T. Ramanathan, "Policy surveillance: a vital public health practice comes of age: table 1," Journal of Health Politics, Policy and Law, vol. 41, no. 6, pp. 1151-1173, 2016.

[31] S. Zeng, D. Luo, C. Zhang, and X. Li, "A correlation-based TOPSIS method for multiple attribute decision making with single-valued neutrosophic information," International Journal of Information Technology \& Decision Making, vol. 19, no. 01, pp. 343-358, 2020.

[32] Z. Yang, X. Li, H. Garg, and M. Qi, "Decision support algorithm for selecting an antivirus mask over COVID-19 pandemic under spherical normal fuzzy environment," International Journal of Environmental Research and Public Health, vol. 17, no. 10, p. 3407, 2020.

[33] N. Zhang, "Analyzing public generated big data and restructuring government decision Making process: review and prospect," Chinese Public Administration, vol. 10, pp. 19-24, 2015.

[34] A. Clarke and H. Margetts, "Governments and citizens getting to know each other open, closed, and big data in public management reform," Policy \& Internet, vol. 6, no. 4, pp. 393-417, 2015.

[35] F. H. Zheng, L. Deng, and J. T. Lin, "Why the performance objectives of subsidy fiscal policy will be aliasing? - based on the third-party performance evaluation of the special fiscal funds from Guangdong province," Journal of Public Management, vol. 3, pp. 122-134+159, 2016.

[36] R. Michels, Political Party, Free Press, New York, NY, USA, 1968.

[37] W. G. Resh and J. D. Marvel, "Loopholes to load-shed: contract management capacity, representative bureaucracy, and goal displacement in federal procurement decisions," International Public Management Journal, vol. 15, no. 4, pp. 525-547, 2012.

[38] H. Liu, J. N. Wu, and M. M. Xu, "A review of goal deviation and its influence factors from different theoretical perspectives," Journal of Public Administration, vol. 1, pp. 151-171+186, 2016.

[39] J. Liang and L. Langbein, "Performance management, highpowered incentives, and environmental policies in China," International Public Management Journal, vol. 18, no. 3, pp. 346-385, 2015.

[40] S. Zeng, S.-M. Chen, and K.-Y. Fan, "Interval-valued intuitionistic fuzzy multiple attribute decision making based on nonlinear programming methodology and TOPSIS method," Information Sciences, vol. 506, pp. 424-442, 2020.

[41] Z. Yang, T. Ouyang, X. Fu, and X. Peng, “A decision-making algorithm for online shopping using deep-learning-based opinion pairs mining and $\mathrm{q}$-rung orthopair fuzzy interaction Heronian mean operators," International Journal of Intelligent Systems, vol. 35, no. 5, pp. 783-825, 2020.

[42] D. Luo, S. Zeng, and J. Chen, "A probabilistic linguistic multiple attribute decision making based on a new correlation coefficient method and its application in hospital assessment," Mathematics, vol. 8, no. 3, p. 340, 2020.

[43] M. D. Blei, Y. A. Ng, and I. M. Jordan, "Latent dirichlet allocation," Journal of Machine Learning Research, vol. 3, pp. 993-1022, 2003.

[44] L. C. Freeman, "Centrality in social networks conceptual clarification," Social Networks, vol. 1, no. 3, pp. 215-239, 1978.

[45] K. Faust, "Centrality in affiliation networks," Social Networks, vol. 19, no. 2, pp. 157-191, 1997. 